\title{
Fisiopatologia dos distúrbios respiratórios do sono
}

\author{
Pathophysiology of sleep-disordered breathing \\ Luciana de Oliveira Palombini
}

\begin{abstract}
Resumo
O sono representa uma fase na qual o sistema respiratório sofre importantes mudanças que levam a uma maior vulnerabilidade e maior chance de ocorrência de anormalidades, mesmo em indivíduos normais. Na transição da vigília para o sono, existe normalmente um aumento da resistência de via aérea superior e prejuízo de diferentes respostas e reflexos protetores, os quais são eficientes em promover e manter a patência da via aérea superior durante a vigília. Em indivíduos que apresentam fatores de risco, tais como anormalidades anatômicas em via aérea superior, essas mudanças associadas ao sono não conseguem ser compensadas de forma eficaz e, com isso, ocorre uma maior chance de ocorrer um distúrbio respiratório do sono. Os distúrbios respiratórios do sono são caracterizados por diferentes graus de diminuição do espaço das vias aéreas superiores. Essa diminuição tem causas multifatoriais, que incluem anormalidades da anatomia de via aérea superior, alteração da resposta neuromuscular e de alterações em receptores de via aérea superior. Provavelmente muitas alterações funcionais e anatômicas em via aérea superior são de característica genética, tornando o individuo com maior risco quando exposto a determinados fatores ambientais, tais como alergias, e a partir da combinação destes fatores haverá uma maior chance de desenvolver distúrbios respiratórios do sono.
\end{abstract}

Descritores: Transtornos do sono; Síndromes da apneia do sono; Sono/fisiologia.

\begin{abstract}
Sleep is a phase during which the respiratory system undergoes major changes. These changes lead to greater vulnerability and a greater risk of abnormalities, even in normal individuals. In the transition from wakefulness to sleep, there is commonly an increase in upper airway resistance and impairment of various protective responses and reflexes, which are efficient in promoting and maintaining upper airway patency during wakefulness. In individuals who present risk factors, such as anatomical abnormalities in the upper airway, these sleep-related changes cannot be efficaciously compensated, which increases the chances that sleep-disordered breathing will occur. Sleep-disordered breathing is characterized by a reduction in the size of upper airways, although the degree of the reduction varies. This reduction has multifactorial causes, which include anatomical abnormalities in the upper airway, alterations in the neuromuscular response and impairment of receptors in the upper airway. Upper airway functional and anatomical changes are likely to have genetic components, and, therefore, individuals exposed to certain environmental factors, such as allergies, have a greater chance of developing sleep-disordered breathing.
\end{abstract}

Keywords: Sleep disorders; Sleep apnea syndromes; Sleep/physiology.

\section{Introdução}

Os distúrbios respiratórios do sono incluem SAOS e SRVAS. 0 distúrbio descrito inicialmente foi SAOS, e as consequências desse distúrbio já estão bem estabelecidas, como um maior risco para doenças cardiovasculares e metabólicas. (1) Posteriormente, SRVAS foi descrita e ainda existem algumas controvérsias a respeito dos critérios diagnósticos e suas consequências a longo prazo. Ambas as condições são associadas a uma redução do espaço em via aérea superior como fator essencial na fisiopatologia.

Sendo um distúrbio frequente, SAOS é caracterizada pelo colapso recorrente da via aérea faríngea induzido pelo sono, levando a hipoxemia e hipercapnia, com despertares durante o sono que são desencadeados com a função de restabelecer a patência da via aérea superior. ${ }^{(1)}$ Em SRVAS, ocorre um estreitamento da via aérea superior associado a um aumento do esforço respiratório na ausência de apneias ou hipopneias. ${ }^{(2)}$ Ambas as condições são associadas a uma maior colapsabilidade na região da via aérea superior. Durante a vigília, a via aérea é protegida por diversos mecanismos ligados à vigília que mantêm a patência da via aérea faríngea, entre esses, um aumento da atividade de músculos dilatadores faríngeos. Entretanto, durante o sono, esses mecanismos não são suficientes para manter a via aérea aberta, ocorrendo uma diminuição do espaço e, consequentemente, uma maior resistência ao fluxo aéreo. 


\section{Efeitos do sono na respiração}

A respiração é influenciada pelos diferentes estados de sono e vigília. Essas influências derivam principalmente de sistemas em tronco cerebral responsáveis pelo controle da ventilação. Durante a vigília, a patência da via aérea superior é mantida em diferentes posturas. ${ }^{(3)}$ Com o inicio do sono, ocorrem diferentes modificações que afetam essa patência, incluindo mudanças na ativação neuromuscular e na ventilação, assim como aumento na carga mecânica. Ocorre um significativo aumento da resistência da via aérea superior. Essas alterações podem resultar em hipoventilação (caso exista uma perda da resposta reflexa ao aumento da carga em via aérea) ou podem resultar em um aumento reflexo do estímulo à ventilação, com manutenção da ventilação e dos gases sanguíneos. Porém, algum grau de hipoventilação sempre ocorre no início do sono, mesmo em indivíduos normais.

Com o início do sono, ocorrem também mudanças na atividade muscular da via aérea superior. Estudos demonstraram ocorrer uma diminuição da atividade tônica e fásica de certos músculos (genioglosso, gêniohioideo, tensor palatino, elevador palatino e palatoglosso). ${ }^{(4)}$ Essas alterações têm sido associadas à diminuição transitória da ventilação e ao aumento da resistência de via aérea superior. (5) Outras alterações respiratórias observadas no inicio do sono incluem uma redução ou perda do aumento do drive respiratório em resposta ao aumento de carga resistiva(6) e alterações na resposta ao aumento de $\mathrm{CO}_{2} \cdot{ }^{(7)}$

\section{Fisiopatologia do estreitamento de via aérea superior em pacientes com distúrbios respiratórios do sono}

\section{Fatores anatômicos}

A via aérea superior de pacientes com SAOS é menor que a de indivíduos normais, ${ }^{(8-21)}$ principalmente por estreitamento promovido pelas paredes laterais, tornando-as mais circulares, ao invés do formato elíptico laterolateral que se vê nos indivíduos normais. (14) Logo, em contraste com indivíduos normais, os quais apresentam o eixo maior da via aérea faríngea orientado nas dimensões laterais, pacientes com SAOS apresentam esse eixo orientado anteroposteriormente, o que corresponde ao estreitamento lateral em nível crítico. ${ }^{(15)}$ Esse estreitamento ocorre devido a diversos fatores. 0 tecido adiposo parafaríngeo encontra-se aumentado em pacientes com SAOS, ${ }^{(16)}$ mas existem dúvidas quanto ao seu poder de compressão extrínseca. As alterações craniofaciais também podem estar envolvidas nesse estreitamento lateral. Dados revelam que os portadores de SAOS têm maior espessura das paredes laterais da faringe. ${ }^{(17)}$

A faringe tem uma rigidez intrínseca, tornando-se mais suscetível ao colapso com a redução de seu diâmetro. Em pacientes com SAOS, há evidências de que a via aérea superior seja mais complacente do que a dos indivíduos normais, ${ }^{(17)}$ apresentando grandes variações nas suas dimensões, principalmente quando ocorre uma alteração do volume pulmonar. ${ }^{(9)}$

Outros aspectos que podem afetar o lúmen da via aérea superior incluem obesidade, deformidades craniofaciais, posição da mandíbula, tamanho da língua e aumento de adenoides (especialmente em crianças).

Alterações estruturais em pacientes com SAOS, como hipertrofia de amígdalas e adenoides, retrognatia mandibular e variações da estrutura craniofacial, têm sido associadas a um maior risco de desenvolvimento de SAOS, presumivelmente devido ao aumento da colapsabilidade da via aérea superior.

\section{Fatores neuromusculares}

\section{Teoria do balanço de pressões}

As forças que promovem colapso da via aérea superior, as quais incluem pressão intraluminal negativa, são opostas por mecanismos neuroanatômicos e neurais que promovem a dilatação da via aérea superior. Em indivíduos acordados, o balanço é mantido a favor da manutenção da patência. Em indivíduos normais, o estreitamento da via aérea pode ocorrer; porém, as forças dilatadoras opostas são suficientes para manter a patência. Durante o sono, em indivíduos normais, a redução da atividade da via aérea não altera o balanço suficientemente para causar colapso. Entretanto, em pacientes com SAOS, o colapso ocorre porque há um desbalanço entre as forças dilatadoras e constritoras. $^{(14)}$ 
Esta teoria do balanço de pressões é um dos principais mecanismos envolvidos na fisiopatologia dos distúrbios respiratórios do sono.

Em indivíduos normais, a faringe é pérvia com pressão intraluminal ao nível atmosférico e requer valores negativos acentuados para seu fechamento. Já os pacientes com SAOS apresentam pressão positiva de fechamento da faringe ou pressão crítica, isto é, o colapso ocorre mesmo quando a pressão intraluminal é atmosférica. ${ }^{(20)}$ Essa observação demonstra a maior colapsabilidade de via aérea superior em indivíduos com distúrbio respiratório do sono e, com isso, eles têm uma maior chance de estreitamento e colapso.

\section{Modelo da via aérea superior como um tubo colapsável}

A via aérea superior apresenta colapso variável em condições de pressão negativa intraluminal, como a que ocorre durante a inspiração. A via aérea superior se comporta como um tubo colapsável. Esse tubo, como no modelo de resistor de Starling, apresenta um padrão de dependência de fluxo na pressão do tubo, ou seja, o fluxo no tubo inicialmente aumenta com o aumento da pressão. Após um nível crítico, ocorre um plateau no fluxo apesar do aumento da pressão. Esse plateau é o que ocorre na limitação ao fluxo aéreo, ou seja, mesmo com o aumento do esforço respiratório não ocorrerá um aumento proporcional no fluxo aéreo. 0 fluxo máximo observado nesse modelo ocorre na dependência da resistência a esse segmento (resistência nasal) e da pressão do compartimento (tecido ao redor da faringe), referida como pressão crítica de fechamento ou pressão de abertura. ${ }^{(22)} 0$ fator indispensável é uma faringe suscetível ao colapso, seja pelo seu calibre reduzido, seja pela pressão ao redor da via aérea superior aumentada.

Pacientes com SAOS apresentam a pressão do compartimento positiva, causando colapso das vias aéreas superiores, a não ser que a pressão positiva seja aplicada através do nariz (CPAP). ${ }^{(23)}$

Em suma, a obstrução em via aérea superior seria causada por uma pressão transmural, tendendo a colapso, devido a uma pressão positiva ao redor da via aérea superior e uma pressão intraluminal negativa. 0 estreitamento da faringe levaria a um aumento da sua complacência (lei do tubo), a um incremento da velocidade de fluxo e subsequente redução da pressão intraluminal (princípio de Bernoulli). Essa promoveria um maior estreitamento e oclusão total das vias aéreas superiores e maior chance de oclusão.

\section{Fatores associados a alterações de receptores locais}

Durante a inspiração, a via aérea superior é sujeita a pressão negativa gerada pela atividade dos músculos respiratórios. 0 reflexo de pressão negativa da via aérea superior se opõe ao efeito colapsável pela ativação de músculos faríngeos dilatadores através de centros respiratórios centrais. ${ }^{(24)} 0$ aumento da atividade dos músculos dilatadores de via aérea superior pode ser demonstrado através da latência mais curta do que uma resposta voluntária. Lesões em qualquer uma dessas vias podem levar à patogênese do colapso de via aérea superior.

\section{Alterações em receptores sensoriais aferentes}

Existem pelo menos dez ou mais diferentes tipos de receptores sensoriais na via aérea superior. Esses receptores respondem a pressão, drive muscular respiratório, frio, calor, irritantes e várias substâncias químicas. Entre todos os receptores da via aérea superior, os mecanorreceptores são os mais bem estudados.

Os mecanorreceptores da via aérea superior são capazes de responder à pressão da via aérea, fluxo aéreo, temperatura e ao próprio tônus muscular da via aérea superior. ${ }^{(22,25,26)}$ Inputs sensoriais desses mecanorreceptores influenciam o tônus da via aérea superior de maneira reflexa, promovendo assim a patência da via aérea superior. Modelos animais têm demonstrado um aumento da atividade do músculo genioglosso com a geração de pressão negativa na via aérea superior. Essa resposta pode ser bloqueada através da aplicação de anestesia local.(27)

Estudos similares têm sido conduzidos em humanos. Um grupo de autores ${ }^{(28)}$ demonstrou que na via aérea superior de indivíduos normais em vigília e anestesiados com anestésico local ocorre um aumento da resistência ao fluxo faríngeo.

0 término do evento obstrutivo apneico e hipopneico geralmente ocorre com um despertar 
no EEG, geralmente precedido por um aumento do esforço respiratório. 0 despertar no EEG ocorre em resposta à informação respiratória aferente gerada na via aérea superior. Além das alterações em gases sanguíneos mediadas através de quimiorreceptores centrais e periféricos presentes em aferentes no pulmão e na caixa torácica, os mecanorreceptores presentes em via aérea superior podem também contribuir para o despertar pós-apneia. ${ }^{(29)}$

Existem diversos fatores associados ao prejuízo dos mecanorreceptores de via aérea superior em pacientes com SAOS. 0 ronco, através de uma vibração de baixa frequência, pode levar ao edema de estruturas de via aérea superior, levando a um maior estreitamento dessa via. As vibrações associadas ao ronco podem resultar em trauma crônico e, com isso, levar a uma disfunção contrátil, perda da sensibilidade e diminuição da via. Logo, a presença de uma lesão que envolve fibras sensoriais (mecanossensoriais) e motoras (músculos dilatadores faríngeos) nessa região poderá levar ao desenvolvimento de SAOS. Já existem evidências que esse tipo de lesão está presente em pacientes com SAOS. ${ }^{(30-32)}$

\section{Contribuição de fatores neuroventilatórios para SAOS}

Mecanismos relacionados ao controle ventilatório também podem ter um papel importante na modulação da colapsabilidade faríngea durante o sono. Diversos estudos indicam haver uma instabilidade do controle respiratório que pode levar a respiração periódica e que pode contribuir para uma via aérea superior com patência prejudicada (obstrução completa parcial) nos períodos do nadir do ciclo respiratório. ${ }^{(33)}$ Como resultado, a instabilidade do controle respiratório pode contribuir, em alguns indivíduos, ao desenvolvimento de SAOS.

A variabilidade na quimiorresponsividade (respostas a hipóxia e hipercapnia) também parece ser um aspecto envolvido na influência central na fisiopatologia da apneia do sono. A importância real dessas respostas como um aspecto importante nos mecanismos da apneia do sono é um assunto ainda controverso, visto que a anatomia e o controle motor da via aérea superior são os aspectos mais importantes na fisiopatologia de SAOS. ${ }^{(34)}$

\section{Outros fatores}

Outros aspectos também envolvidos na fisiopatologia de SAOS são o volume pulmonar e alterações hemodinâmicas. Existe uma associação entre o volume pulmonar e o tamanho da via aérea superior, descrito em humanos e em animais, que demonstram um aumento no espaço da via aérea superior com volumes pulmonares maiores. ${ }^{(35)}$ Esse efeito é provavelmente devido ao estiramento da traqueia, levando a uma via aérea superior maior e a uma diminuição da resistência ao fluxo aéreo. ${ }^{(36)}$ Os pacientes com SAOS apresentam um aumento nesse efeito, ou seja, uma maior mudança nas dimensões da via aérea superior com mudanças do volume pulmonar. ${ }^{(37)} \mathrm{Em}$ indivíduos normais, ao fim da expiração ocorre uma redução do calibre da via aérea superior, mecanismo mais acentuado em pacientes com SAOS.(7) 0 oposto, ou seja, a dilatação, ocorre na inspiração mediante a tração caudal das vias aéreas superiores pelo aumento do volume na caixa torácica resultante da inspiração. Tendo os pacientes com SAOS uma faringe mais complacente, eles são, portanto, mais dependentes desse último mecanismo. ${ }^{(38)}$

Outro aspecto que tem sido relatado como um possivel fator para SAOS são as alterações dinâmicas que ocorrem em via aérea superior. Essas envolvem a vasodilatação no pescoço, que pode aumentar a resistência da via aérea superior. Em pacientes com SAOS acordados, esse efeito foi observado quando os membros inferiores foram elevados. ${ }^{(39)}$ Esse mecanismo pode também ser potencializado no sono ou em estados hipervolêmicos.

\section{Fatores genéticos na fisiopatologia dos distúrbios respiratórios do sono}

Existe uma interação entre fatores genéticos e ambientais para o desenvolvimento dos distúrbios respiratórios do sono. Estudos têm enfatizado o fato de que SAOS é uma doença familiar. ${ }^{(40)}$ No entanto, a presença de agregados familiares não implica necessariamente na presença de fatores genéticos. Provavelmente existem genes que controlam o desenvolvimento craniofacial associados a um maior risco de ocorrer anormalidades respiratórias durante 0 sono, pois características relacionadas a uma via aérea pequena estão presentes em famílias com distúrbios respiratórios do sono. ${ }^{(41)}$ Outro 
aspecto genético envolvido no desenvolvimento de SAOS está relacionado à quimiossensibilidade para resposta a hipóxia e hipercapnia. Uma resposta genética específica pode levar a uma maior vulnerabilidade à resposta inadequada a hipóxia e hipercapnia. ${ }^{(42,43)}$

Entre os fatores ambientais que podem combinar com aspectos genéticos para 0 desenvolvimento dos distúrbios respiratórios do sono, podem-se citar alergias, infecções e o aumento anormal do tecido linfoide localizado na região (amígdala e adenoide), o qual pode ser influenciado por alergias e infecções. 0 efeito da respiração nasal no desenvolvimento das estruturas faciais foi demonstrado em um estudo. (44) 0 aumento da resistência nasal foi induzido experimentalmente através da introdução de cones na narina de macaco rhesus. 0 aumento da resistência levou à marcada diminuição no crescimento mandibular e ao desenvolvimento facial anormal. Esse foi revertido após o restabelecimento da respiração nasal normal.

Esse efeito pode ser observado também em crianças que apresentam respiração bucal. Essas crianças apresentam uma maior resistência nasal e podem também apresentar aumento das adenoides. Alergias de vias aéreas superiores e infecção, assim como adenoides e amígdalas hipertrofiadas, levam a uma resistência nasal anormal. 0 aumento da resistência pode levar a um desenvolvimento faríngeo e facial anormal que irá afetar vários músculos, incluindo língua e masseter. Essa disfunção muscular terá impacto no crescimento maxilomandibular e no posicionamento do osso hioide. Resumindo, o desenvolvimento facial anormal representa um fator de risco para via aérea pequena, a qual representa um fator de risco para o desenvolvimento dos distúrbios respiratórios do sono. 0 reconhecimento precoce dos distúrbios respiratórios do sono deve sempre ser feito em crianças, pois o tratamento nesta fase pode permitir um desenvolvimento normal da via aérea superior e, com isso, prevenir o desenvolvimento de SAOS na idade adulta.

\section{Considerações finais}

Em resumo, indivíduos que apresentam distúrbios respiratórios do sono possuem uma predisposição anatômica em fase precoce, e essa predisposição, em combinação com diferentes fatores ambientais, irá levar a anormalidades respiratórias durante o sono, entre elas SAOS e SRVAS. Os distúrbios respiratórios do sono são associados a um estreitamento da via aérea superior o qual não é compensado pelos mecanismos de compensação presentes. Estes distúrbios desenvolvem-se a partir da combinação de diferentes aspectos em mecanismos e respostas do sistema respiratório, entre estes, alterações anatômicas, alterações em receptores locais, alterações na resposta cortical e na função neuromuscular e anormalidades do controle ventilatório. 0 reconhecimento precoce na infância das anormalidades é importante, pois o tratamento destas nesta fase é a única maneira de realizar a prevenção dos distúrbios respiratórios do sono do adulto.

\section{Referências}

1. Sleep-related breathing disorders in adults: recommendations for syndrome definition and measurement techniques in clinical research. The Report of an American Academy of Sleep Medicine Task Force. Sleep. 1999;22(5):667-89.

2. Guilleminault C, Stoohs R, Clerk A, Cetel M, Maistros P. A cause of excessive daytime sleepiness. The upper airway resistance syndrome. Chest. 1993;104(3):781-7.

3. Badr MS. Pathophysiology of upper airway obstruction during sleep. Clin Chest Med. 1998;19(1):21-32.

4. Badr MS, Skatrud JB, Dempsey JA, Begle RL. Effect of mechanical loading on expiratory and inspiratory muscle activity during NREM sleep. J Appl Physiol. 1990;68(3):1195-202.

5. Wiegand DA, Latz B, Zwillich CW, Wiegand L. Upper airway resistance and geniohyoid muscle activity in normal men during wakefulness and sleep. J Appl Physiol. 1990;69(4):1252-61.

6. Tangel DJ, Mezzanotte WS, White DP. Influence of sleep on tensor palatini EMG and upper airway resistance in normal men. J Appl Physiol. 1991;70(6):2574-81.

7. Wiegand L, Zwillich CW, White DP. Collapsibility of the human upper airway during normal sleep. J Appl Physiol. 1989;66(4):1800-8.

8. Henke KG, Dempsey JA, Badr MS, Kowitz JM, Skatrud JB. Effect of sleep-induced increases in upper airway resistance on respiratory muscle activity. J Appl Physiol. 1991;70(1):158-68.

9. Bohlman ME, Haponik EF, Smith PL, Allen RP, Bleecker ER, Goldman SM. CT demonstration of pharyngeal narrowing in adult obstructive sleep apnea. AJR Am J Roentgenol. 1983;140(3):543-8.

10. Dempsey JA, Skatrud JB, Jacques AJ, Ewanowski SJ, Woodson BT, Hanson PR, et al. Anatomic determinants of sleep-disordered breathing across the spectrum of clinical and nonclinical male subjects. Chest. 2002;122(3):840-51.

11. Issa FG, Sullivan CE. Upper airway closing pressures in obstructive sleep apnea. J Appl Physiol. 1984;57(2):520-7. 
12. Remmers JE, deGroot WJ, Sauerland EK, Anch AM. Pathogenesis of upper airway occlusion during sleep. $J$ Appl Physiol. 1978;44(6):931-8.

13. Haponik EF, Smith PL, Bohlman ME, Allen RP, Goldman SM, Bleecker ER. Computerized tomography in obstructive sleep apnea. Correlation of airway size with physiology during sleep and wakefulness. Am Rev Respir Dis. 1983;127(2):221-6.

14. Rivlin J, Hoffstein V, Kalbfleisch J, McNicholas W, Zame $\mathrm{N}$, Bryan AC. Upper airway morphology in patients with idiopathic obstructive sleep apnea. Am Rev Respir Dis. 1984;129(3):355-60.

15. Rodenstein DO, Dooms G, Thomas Y, Liistro G, Stanescu DC, Culée C, et al. Pharyngeal shape and dimensions in healthy subjects, snorers, and patients with obstructive sleep apnoea. Thorax. 1990;45(10):722-7.

16. Horner RL, Mohiaddin RH, Lowell DG, Shea SA, Burman ED, Longmore DB, et al. Sites and sizes of fat deposits around the pharynx in obese patients with obstructive sleep apnoea and weight matched controls. Eur Respir J. 1989;2(7):613-22.

17. Rivlin J, Hoffstein V, Kalbfleisch J, McNicholas W, Zamel $\mathrm{N}$, Bryan AC. Upper airway morphology in patients with idiopathic obstructive sleep apnea. Am Rev Respir Dis. 1984;129(3):355-60.

18. Brown IG, Bradley TD, Phillipson EA, Zamel N, Hoffstein V. Pharyngeal compliance in snoring subjects with and without obstructive sleep apnea. Am Rev Respir Dis. 1985;132(2):211-5.

19. Suratt PM, McTier RF, Wilhoit SC. Collapsibility of the nasopharyngeal airway in obstructive sleep apnea. Am Rev Respir Dis. 1985;132(5):967-71.

20. Isono S, Remmers JE. Anatomy and physiology of upper airway obstruction. In: Kryger MH, Roth T, Dement WC, editors. Principles and practice of sleep medicine. Philadelphia: Saunders; 1994. p. 632-56.

21. Gleadhill IC, Schwartz AR, Schubert N, Wise RA, Permutt S, Smith PL. Upper airway collapsibility in snorers and in patients with obstructive hypopnea and apnea. Am Rev Respir Dis. 1991;143(6):1300-3.

22. Mathew OP, Sant'Ambrogio G, Fisher JT, Sant'Ambrogio FB. Laryngeal pressure receptors. Respir Physiol. 1984;57(1):113-22.

23. Morrison DL, Launois SH, Isono S, Feroah TR, Whitelaw WA, Remmers JE. Pharyngeal narrowing and closing pressures in patients with obstructive sleep apnea. Am Rev Respir Dis. 1993;148(3):606-11.

24. Smith PL, Wise RA, Gold AR, Schwartz AR, Permutt S. Upper airway pressure-flow relationships in obstructive sleep apnea. J Appl Physiol. 1988;64(2):789-95.

25. Hwang JC, St John WM, Bartlett D Jr. Receptors responding to changes in upper airway pressure. Respir Physiol. 1984;55(3):355-66.

26. Sant'Ambrogio G, Mathew OP, Fisher JT, Sant'Ambrogio FB. Laryngeal receptors responding to transmura pressure, airflow and local muscle activity. Respir Physiol. 1983;54(3):317-30.

27. Mathew OP, Abu-Osba YK, Thach BT. Influence of upper airway pressure changes on genioglossus muscle respiratory activity. J Appl Physiol. 1982;52(2):438-44.
28. DeWeese EL, Sullivan TY. Effects of upper airway anesthesia on pharyngeal patency during sleep. J Appl Physiol. 1988;64(4):1346-53.

29. McNicholas WT, Coffey M, McDonell T, O’Regan R, Fitzgerald MX. Abnormal respiration during sleep in normal subjects following selective topical oropharyngeal and nasal anesthesia. Am Rev Respir Dis. 1987;135:1316-19.

30. Issa FG, McNamara SG, Sullivan CE. Arousal responses to airway occlusion in sleeping dogs: comparison of nasal and tracheal occlusions. J Appl Physiol. 1987;62(5):1832-6.

31. Friberg D, Ansved T, Borg K, Carlsson-Nordlander B, Larsson H, Svanborg E. Histological indications of a progressive snorers disease in an upper airway muscle. Am J Respir Crit Care Med. 1998;157(2):586-93.

32. Friberg D, Gazelius B, Lindblad LE, Nordlander B. Habitual snorers and sleep apnoics have abnormal vascular reactions of the soft palatal mucosa on afferent nerve stimulation. Laryngoscope. 1998;108(3):431-6.

33. Kimoff RJ, Sforza E, Champagne V, Ofiara L, Gendron D. Upper airway sensation in snoring and obstructive sleep apnea. Am J Respir Crit Care Med. 2001;164(2):250-5.

34. Hudgel DW, Chapman KR, Faulks C, Hendricks C. Changes in inspiratory muscle electrical activity and upper airway resistance during periodic breathing induced by hypoxia during sleep. Am Rev Respir Dis. 1987;135(4):899-906.

35. Dempsey JA, Smith CA, Przybylowski T, Chenuel B, Xie $\mathrm{A}$, Nakayama $\mathrm{H}$, et al. The ventilatory responsiveness to $\mathrm{CO}(2)$ below eupnoea as a determinant of ventilatory stability in sleep. J Physiol. 2004;560(Pt 1):1-11.

36. Sériès F, Cormier $Y$, Desmeules M. Influence of passive changes of lung volume on upper airways. J Appl Physiol. 1990;68(5):2159-64.

37. Van de Graaff WB. Thoracic influence on upper airway patency. J Appl Physiol. 1988;65(5):2124-31.

38. Hoffstein V, Zamel N, Phillipson EA. Lung volume dependence of pharyngeal cross-sectional area in patients with obstructive sleep apnea. Am Rev Respir Dis. 1984;130(2):175-8.

39. Burger CD, Stanson AW, Daniels BK, Sheedy PF 2nd, Shepard JW Jr. Fast-CT evaluation of the effect of lung volume on upper airway size and function in normal men. Am Rev Respir Dis. 1992;146(2):335-9.

40. Seelagy MM, Schwartz AR, Russ DB, King ED, Wise RA, Smith PL. Reflex modulation of airflow dynamics through the upper airway. J Appl Physiol. 1994;76(6):2692-700.

41. Manon-Espaillat R, Gothe B, Adams N, Newman C, Ruff R. Familial 'sleep apnea plus' syndrome: report of a family. Neurology. 1988;38(2):190-3.

42. Mathur R, Douglas NJ. Family studies in patients with the sleep apnea-hypopnea syndrome. Ann Intern Med. 1995;122(3):174-8.

43. Redline S, Leitner J, Arnold J, Tishler PV, Altose MD. Ventilatory-control abnormalities in familial sleep apnea. Am J Respir Crit Care Med. 1997;156(1):155-60.

44. Vargervik K, Miller AJ, Chierici G, Harvold E, Tomer BS. Morphologic response to changes in neuromuscular patterns experimentally induced by altered modes of respiration. Am J Orthod. 1984;85(2):115-24.

\section{Sobre a autora}

\section{Luciana de Oliveira Palombini}

Médica Pneumologista e Coordenadora do Instituto do Sono de São Paulo, São Paulo (SP) Brasil.

E-mail para contato: Ipalombini@hotmail.com (L. Palombini) 